\title{
Quark degrees of freedom in hadronic systems
}

\author{
Vicente Vento ${ }^{\text {a* }}$
}

${ }^{a}$ Departamento de Física Teórica - IFIC, Universidad de Valencia - CSIC, E-46100 Burjassot (Valencia), Spain

Quantum Chromodynamics $(Q C D)$ is the theory of the strong interactions. We review descriptions of hadronic systems motivated by $Q C D$, analyzing the recent controversy between gluonic and bosonic degrees of freedom under the prism of the Cheshire Cat Principle. Our analysis leads to an optimal scheme to study hadronic properties. We proceed to extend this low energy descriptions to the deep inelastic regime.

\section{Introducing Quantum Chromodynamics}

There is little doubt nowadays in the physics community that Quantum Chromodynamics $(Q C D)$ [1,2] is the theory that describes the hadronic interactions within the Standard Model. $Q C D$ is a renormalizable quantum field theory whose elementary fields are quarks, spin $\frac{1}{2}$ fermions with color $(A=1,2,3)$ and flavor $(i=1, \ldots, 6)$, and gluons, spin 1 bosons with color $(a=1, \ldots, 8)$, coupled to achieve $S U_{\text {color }}(3)$ gauge invariance of the theory,

$$
\mathcal{L}=i \bar{\Psi}_{i}^{A}(x) \gamma^{\mu}\left(D_{\mu}\right)_{A B} \Psi(x)_{i}^{B}-M_{i j} \bar{\Psi}_{i}(x) \Psi_{j}(x)-\frac{1}{4} F^{\mu \nu a} F_{\mu \nu}^{a}+\text { gauge fixing + ghosts. (1) }
$$

The quark fields have been denoted by $\Psi_{j}^{A}$, the covariant derivative is given by

$$
\left(D_{\mu}\right)_{A B}=\delta_{A B} \partial_{\mu}-i g A_{\mu}^{a}\left(t^{a}\right)_{A B}
$$

where $t^{a}$ are the group generators in the quark representation satisfying the algebra of the group, $\left[t_{a}, t_{b}\right]=f_{a b c} t_{c}, A_{\mu}^{a}$ denote the gluon fields and $g$ the coupling constant. The dynamics provides self-couplings for the gluons as can be seen from the definition of the gluonic tensor,

$$
F_{\mu \nu}^{a}=\partial_{\mu} A_{\nu}^{a}-\partial_{\nu} A_{\mu}^{a}+g f_{a b c} A_{\mu}^{b} A_{\nu}^{c} .
$$

Finally the only dynamics associated with flavor comes from the mass matrix,

$$
M_{i j}=m_{i} \delta_{i j}
$$

a parameter set whose origin is external to the theory.

Despite its apparent simplicity, almost thirty years have passed since its original formulation, and we have not yet been able to solve it exactly. However much has been learned about its structure and its properties.

${ }^{*}$ The work reported here was supported by Spain DGCYT-PB97-1127 and the European Union ERB FMRX-CT96-008. 


\subsection{Exact Results}

There has been progress in understanding the properties of strongly interacting gauge theories through the development of rigorous inequalities. In particular a surprising amount of information about symmetry realization in $Q C D$ follows from relatively simple facts about fermion determinants and propagators in Euclidean space-time [3] and anomaly constraints [4]:

i) the pion mass is the smallest among the mesons;

ii) the pion mass is smaller than any baryon mass;

iii) if axial currents are conserved the axial symmetry is spontaneously broken;

iv) if vector currents are conserved the vector symmetry is unbroken;

v) parity is not spontaneously broken [5].

These exact results confirm that $Q C D$ verifies the experimental observation that chiral symmetry is realized in the hadronic interactions à la Goldstone, i.e. in spontaneously broken fashion, and that the pion is the lightest hadron in nature. Thus, even in the massless quark limit, the $Q C D$ vacuum breaks chiral symmetry despite the fact that the currents derived from the $Q C D$ lagrangian are conserved.

\subsection{Asymptotic freedom}

A very important property of $Q C D$, known as asymptotic freedom, is that the effective coupling constant vanishes at short distances [6]. This can be mathematically expressed, in perturbation theory to leading order, by the so called running coupling constant

$$
\alpha_{S}\left(Q^{2}\right)=\frac{12 \pi}{\left(33-2 n_{f}\right) \log \left(\frac{Q^{2}}{\Lambda^{2}}\right)} .
$$

A mathematical statement that implies that at large spacelike momenta $\left(Q^{2} \rightarrow \infty\right)$ the theory behaves as a free field theory modulo logarithmic corrections. $\Lambda$ introduces the scale for perturbative phenomena in the theory. Moreover for sufficiently large momenta the corrections to the free field theory can be calculated in a power series in $\alpha_{S}\left(Q^{2}\right)$. The contributions to the physical processes are determined by the elementary vertices determined from the lagrangian and the renormalization group.

These features can be seen directly by looking at high energy Jets [7] and have been proven extremely successful in the interpretation of the Deep Inelastic lepton-hadron Scattering (DIS) data, what is known as Bjorken scaling and the logarithmic deviations from it 8 .

\subsection{Confinement}

How does the theory behave away from the asymptotic regime? We have not been able to isolate quarks and gluons in the laboratory. This could have been taken as a failure of $Q C D$, however it has turned into the dynamical principle which governs the low energy behavior of the theory known as confinement. The fact that $\alpha\left(Q^{2}\right)$ grows for low momenta (see Eq. (5)) is taken as an indication that quark and gluons are confined. However the 
theory becomes in that regime highly non-perturbative and one cannot use perturbative technology to describe this dynamics. Does confinement arise from the quantum theory obtained from Eq(1)? We have not been able to answer this question exactly except in particular situations: lower dimensionality [9,10], Supersymmetric Yang Mills theories [11] and in the most promising approximation to the theory, i.e. Lattice QCD, for pure glue [12].

The problem with real QCD is that the structure of the vacuum is highly non trivial. It is precisely the vacuum properties which dominate the low energy behavior. All types of non perturbative field configurations inhabit the groundstate, instantons, merons, monopoles,..., and moreover seem to play a role in the way the theory is realized at the hadronic level.

Let us present a plausible confinement scenario [13]. The effective color interaction becomes stronger as the separation between the probe color charges increases. In this regime a new phenomenon takes place. When the distance becomes larger than some number times $\frac{1}{\Lambda}$, and exceeds a critical one, the branching of the gluons becomes so intensive that one cannot speak about individual gluons, but rather should describe the interaction in terms of chromoelectric and chromomagnetic fields. In $Q C D$, the chromoelectric field between the probe charges squeezes into a sausage-like configuration, the flux tube. This situation is reminiscent of the Meissner effect in superconductivity. We deal here with chromoelectric flux-tubes, i.e. with a Dual Meissner effect. Confinement is insured by a condensation of magnetic monopoles (not charges), which force the fields to form the flux tubes in the $Q C D$ vacuum.

Nobody has ever proved this scenario. There are however indications arising from lattice- $Q C D$ calculations [14] and from N=2 SUSY Yang Mills [15] where in the strong coupling regime monopoles have been shown to condense.

\subsection{Chiral symmetry}

In the massless quark limit $Q C D$ is invariant under $S U\left(n_{f}\right) \otimes S U\left(n_{f}\right)$ chiral symmetry. This symmetry is however spontaneously broken and for two flavors the pion is the associated Goldstone boson. Microscopically the properties of the vacuum are crucial in describing the realization of chiral symmetry. The Casher-Banks formula 16,

$$
<\bar{q} q>=-\pi \rho(\lambda=0)
$$

tells us that the condensate, namely the order parameter for the transition from the unbroken to the spontaneously broken phase, is connected with the density $(\rho)$ of quark states of small virtuality $(\lambda)$. It has been shown [17] that the instantons play an instrumental role in explaining microscopically the realization of chiral symmetry.

\subsection{Imitating $Q C D$}

The main effort in understanding $Q C D$ at present, since an exact solution seems non atainable, is into developing its lattice approximation. However this approach is still very primitive in order to produce, without guidance, realistic results. It may serve to support or reject some of the scenarios that may be proposed in view of the data, but as of yet is still far away from being able to predict or explain many of the hadronic properties [12].

Physicists have turned to other means of understanding the data by approximating the theory. The effective theory approach has been widely used for describing low en- 
ergy data. In this method one starts from the original action and measure of the theory, integrates out the irrelevant degrees of freedom and using the appropriate matching conditions obtains a different, much more tractable, field theory with the same S-matrix [18]. This procedure has been used with notable precision at high energies. At low energies we are not able to follow strictly the procedure and therefore apply a celebrated, though unproven, theorem by Weinberg [19], which states that one should write the most general lagrangian, constructed from the accesible degrees of freedom in the domain under consideration, which satisfies the relevant symmetries of the theory. This is the scheme used for Chiral Perturbation Theory [20], which has been extremely successful in low energy meson physics.

A second procedure has been the use of approximation methods to truncate $Q C D$. The most relevant examples are $\frac{1}{N}$ [21] and HQET 222]. In this case we proceed in terms of an expansion parameter $\frac{1}{N}, N$ being the number of colors for the former, and $\frac{1}{M}, M$ being the mass of the heavy quark, for the latter. Unluckily we have been only able to develop arguments in the $\frac{1}{N}$ case since, despite many efforts, we have not been able to sum the planar graphs for $Q C D$. HQET has been extremely successful in discovering new spin symmetries for the heavy quark sector and describing decays in terms of only a few parameters.

Lastly, people have resorted to use their imagination to produce models, whose relation with the theory is at most qualitative, but which are extremely predictive once they are fully developed. The basic assumption is that one knows the way the theory is realized at low energies, i.e., the vacuum, the Fock states and the dynamics among the constituents. There are many models, which are becoming more detailed following the advances in our microscopic understanding of the properties of the theory.

The subject of my discussion will be the physics associated with models of hadron structure, but before I proceed along these lines let me mention an example of how specific features of the microscopic structure of the theory can motivate explanations for particular phenomena. The instantons building the $Q C D$ vacuum induce interactions among the quarks which have been shown to be responsible for many observable effects of hadron physics, both in the vacuum and in the nuclear medium 17,23,24.

It has become clear by now that it is hard to make definitive statements about hadrons made up of light quarks ( $\mathrm{u}, \mathrm{d}$ and $\mathrm{s}$ ), were the nonperturbative structure of the theory is difficult to avoid. Nevertheless the hadron spectrum and the interactions among the hadrons display regularities which, when properly formulated, correlate with simple properties of the fundamental theory. They are best expressed by inventing mechanisms that describe the undisclosed features of the theory.

\section{Modelling hadron structure}

The role of models in $Q C D$ is to produce simple physical pictures that connect the phenomenological regularities with the underlying structure. Models should be used to

guide fundamental calculations and experiments. They are not substitute for the theory and therefore should be closely tied to it and abandoned when they become a divertimento by themselves.

Historically the first models of hadron structure appeared in the sixties right after Gell- 
Mann and Zweig [25] introduced the Quarks. Dalitz in his beautiful Les Houches Lectures of 1965 [26], performed a phenomenological analysis leading to flavor forces of the type

$$
\begin{aligned}
U(\bar{q} q)= & U_{p 1}\left(1-\overrightarrow{\sigma_{1}} \cdot \overrightarrow{\sigma_{2}}\right)\left(1-\tilde{F}_{1} \cdot \tilde{F}_{2}\right)+U_{p 8}\left(1-\overrightarrow{\sigma_{1}} \cdot \overrightarrow{\sigma_{2}}\right)\left(8+\tilde{F}_{1} \cdot \tilde{F}_{2}\right)+ \\
& U_{v 1}\left(3+\overrightarrow{\sigma_{1}} \cdot \overrightarrow{\sigma_{2}}\right)\left(1-\tilde{F}_{1} \cdot \tilde{F}_{2}\right)+U_{v 8}\left(3+\overrightarrow{\sigma_{1}} \cdot \overrightarrow{\sigma_{2}}\right)\left(8+\tilde{F}_{1} \cdot \tilde{F}_{2}\right)
\end{aligned}
$$

where $\vec{\sigma}$ arises from the quark spin generators and $\tilde{F}$ from the $\mathrm{SU}(3)$ flavor generators. This most general Ansatz produced a beautiful fit, which had to be dropped because no explanation was found to justify the abandonment of the Spin-Statistics theorem. The introduction of color [27] solved all these problems and led to the formulation of $Q C D$.

The Quark Model was revisited soon after the introduction of $Q C D$ by De Rújula, Georgi and Glashow [28], who developed a scheme to incorporate color to the spectroscopic analysis. Their philosophy has motivated many of the developments thereafter. In particular the Bag Model [29] relies on a quantum field theoretic description to implement the same philosophy. The aim of these approaches is to define a scheme which incorporates the properties of $Q C D$ as we next describe.

\subsection{Confinement}

The models contain a mechanism which provides the dynamics to bind quarks (antiquarks) to form color singlet hadrons. These mechanisms are varied: color independent attractive potentials [28,30], field boundary conditions providing the confinement of color to a space-time tube [29], etc.

\subsection{Asymptotic freedom}

Guided by the apparent absence of strong renormalization (higher twist effects) in DIS it was thought that once confinement is implemented a picture of a hadron arises where perturbative $Q C D$ is qualitatively reliable. Therefore an additional residual interaction between the quarks based on the One Gluon Exchange contribution was introduced. This so called color-magnetic interaction has an operator structure in color space of the form

$$
\sum_{i \neq j} F\left(r_{i j}\right) \lambda_{i} \cdot \lambda_{j} \overrightarrow{\sigma_{i}} \cdot \overrightarrow{\sigma_{j}}
$$

which has been implemented in all approaches and is responsible among other things for the $\Delta$-Nucleon mass splitting. With the proper simplifications, taking into account the Fock Space of the lowest quark states, it can be reduced to an interaction of flavor type [31] which reflects the strong flavor dependence explicit in the original parametrization of Dalitz.

Models satisfying these two properties have been extremely successful in explaining a large amount of data [30,31]. They have been used as low energy descriptions of $Q C D$, and by means of the Operator Product Expansion, we have been able to study and predict high energy data [32, 33]. The so called proton spin problem can be understood as a transition from constituent quarks to currents quarks, antiquarks and gluons. Moreover it has been shown that the data serve to unveil the Regge structure of the constituent quarks [33. Moreover they have also been used to speculate about the behavior of hadrons in the nuclear medium [34]. 
Despite the many successes of the schemes just described, they all lack an ingredient, namely the realization of a fundamental symmetry of $Q C D$, i.e. spontaneously broken chiral symmetry. The role of the pseudoscalar mesons as Goldstone boson was not incorporated in these models and the pion is considered a conventional $\bar{q} q$ boundstate. This absence implied that all traditional low energy hadron physics, dominated by chiral flavor symmetry, had to appear from confined perturbative $Q C D$. Although the static properties were well reproduced, except in the pseudoscalar meson sector, whose mass should vanish in the massless quark limit, the attempts to reproduce the long distance properties of the hadronic interaction failed.

\subsection{Chiral symmetry}

Chiral symmetry was implemented initially in the bag model formalism since the field theoretic language was more suitable for the task. Confined perturbative $Q C D$ was kept in the interior of the bag, while in the outside region an elementary meson field was introduced by means of a non linear sigma model. The dynamics between the quarks and the pions arises naturally from implementing chiral current conservation at the boundary [35]. Soon thereafter attempts were made to implement this scheme into the potential models [36].

These models are able to reproduce the static properties also with great precision. Moreover the appearence of the Goldstone modes allows them to describe without further ingredients low energy properties associated with chiral symmetry in a straightforward fashion. Let us describe two lines of thought much developed in these days.

\subsubsection{The Cheshire Cat Principle}

In two dimensions fermion theories are exactly bosonizable. Let us separate space arbitrarily in two regions. The left hand side we describe in terms of a fermion theory, e.g. $Q C D$ and the right hand side in terms of the equivalent bosonic theory. If the appropriate boundary conditions are defined, by matching both classical current conservation and quantum mechanical anomalies, the Cheshire Cat Principle (CCP) states: the physical properties have to be independent of the position of the boundary [37. In four dimensions the situation can be best appreciated in a bag picture. Inside the bag we have confined perturbative $Q C D$ and outside the equivalent bosonic theory, where solitons, i.e., skyrmions, carry the baryon number. The CCP states that the physical observables have to be radius independent [38]. $Q C D$ in four dimensions is not exactly bosonizable nor it is solvable to any degree of approximation in the quark gluon language, thus the CCP principle can be only approximate. We have used the opposite logic. We have taken the CCP as a quality control check over the approximations involved [38]: only for those values of the radius where there appears an almost radius independence can the approximations be trusted. Monotonic functions of the radius for the observables imply that important contributions are missing. Many observables have been analyzed successfully under this philosophy. It is important to stress that not all the observables require the same approximations. Those observables, where anomalies contribute, like in the spin problem, are very delicate to calculate, because no contribution is dominant and large cancellations among all of them occur [39]. 


\subsubsection{Chiral quarks}

Manohar and Georgi tried to understand the successes of the non-relativistic quark model in terms of effective theories 40]. Their main argument is that the scale associated with confinement $\Lambda_{Q C D}(\approx 100-300 \mathrm{MeV})$ is smaller than that associated with chiral symmetry breaking $\Lambda_{\chi S B}(\approx 1000 \mathrm{MeV})$. Therefore there is a region of momentum where quarks, gluons and pions coexist. Using the effective theory approach they obtain a theory where quarks and gluons interact by means of the conventional color couplings while quarks and pions through a non-linear sigma model. The quark and gluon fields are effective fields, i.e., the latter with a constituent mass obtained from chiral symmetry breaking. By naive dimensional arguments and by properly matching the unknown coupling constants of the theory they get from the hyperfine splitting $\alpha_{s} \approx 0.3$ and from $\pi-\pi$ scattering the chiral scale $\Lambda_{\chi S B}=4 \pi f_{\pi}$, where $f_{\pi} \approx 93 \mathrm{MeV}$ is the pion decay constant. They also show that gluon loops are suppresed as compared to quark and pion loops, a statement which is much dependent on their dimensional arguments and matching scheme.

Let me conclude this section by stating that the implementation of chiral symmetry both in bag model schemes and in effective theories leads to a theory which contains quarks, gluons and Goldstone mesons as the natural degrees of freedom to describe the low energy properties. I will not support therefore any of the sides of the recent IsgurGlozman [1, 42] debate but conclude that both extremes are unnatural. As shown very clearly in the investigations on the CCP a full quark gluonic theory would imply the consideration of higher order contributions making the calculation impractical, while a full mesonic theory would need ultimately the inclusion of many mesons, not only making the calculation impractical, but filled with parameters to be fitted. In my opinion certain features of the structure of hadrons appear in an extremely simple manner in terms of the chromomagnetic interaction [31], while others, in particular the long range tale of the nucleon-nucleon interaction are easily described by the pion [35]. A scheme which incorporates both features, like the Chiral Bag Model or the Chiral Quark Model, is most appropriate to develop a model due to its simplicity.

\section{Applications}

I proceed to motivate and review some of the developments surrounding my own research. The investigation aims at testing models of $Q C D$ in different regimes and circumstances to show what they teach us about the true theory and analyze if they are helpful in predicting new data.

\subsection{Approximate bosonization}

The $C C P$ has been tested in many instances with notable success [37]. It has been observed at the level of topological quantities, i.e., baryon charge fractionation [45,46] and approximately at the level of non topological observables, i.e., masses, magnetic moments, etc ... 39,47]. The explicit manifestation of the $C C P$ in the latter is through some type of minimum sensitivity principle in terms of the bag radius. Moreover the mean value about which observables are not sensitive to the radius corresponds to the confinement scale $R \sim \frac{1}{\Lambda_{Q C D}}$. 
There is one case, the flavor axial singlet charge $(F S A C)$, were its implementation had not been successful beyond doubt and therefore it has merit our attention [39]. From the phenomenological point of view the $F S A C$ is associated with the $\eta^{\prime}$ and therefore with the anomaly [9]. This observable is relevant for what has been referred to as the proton spin problem. In the chiral bag model the formulation is very elaborate. Confinement induces through quantum effects a color anomaly, which leads to a surface coupling of the $\eta^{\prime}$ with the gluon field. The latter induces a gauge non invariant Chern-Simons current, whose expectation value we need to calculate. We have shown that the presence of the surface term generated by the proper matching of the color anomaly with the surface gluon- $\eta^{\prime}$ coupling allows us to obtain for this observable a value close to the data over a wide range of bag radii. The CCP was instrumental in obtaining this agreement [39].

\subsection{Chiral quarks}

We have seen that in an effective theory approach the light chiral quarks appear if $\Lambda_{\chi S B}>>\Lambda_{Q C D}$. In the region between the two scales we have quarks and gluons interacting through the $S U(3)$ color interaction. Since $S U(3) \otimes S U(3)$ global chiral symmetry is spontaneously broken, there must be also an octet of Goldstone bosons which are introduced in the effective theory also as fundamental fields. All other hadrons are obtained as $q q q$ or $\bar{q} q$ bound sates.

We shall use for effective lagrangian below the chiral scale a linear realization, although non-linear realizations can be immediatly developed, i.e.

$$
\begin{aligned}
\mathcal{L}= & i \bar{\Psi}_{i}^{A}(x) \gamma^{\mu}\left(D_{\mu}\right)_{A B} \Psi(x)_{i}^{B}-M_{i j} \bar{\Psi}_{i}(x) \Psi_{j}(x)-\frac{1}{4} F^{\mu \nu a} F_{\mu \nu}^{a}+ \\
& \bar{\Psi}_{i}(x)\left(\sigma \delta_{i j}+i \gamma_{5} \vec{\pi} \cdot \vec{\tau}_{i j}\right) \Psi_{j}(x)+\frac{1}{2} \partial_{\mu} \sigma \partial^{\mu} \sigma+\frac{1}{2} \partial_{\mu} \vec{\pi} \cdot \partial^{\mu} \vec{\pi} .
\end{aligned}
$$

This is the fundamental support for many of the chiral quark models. The quarks here are effective quarks and therefore the masses appearing in the lagrangian are constituent masses.

Our aim has been to approach phenomenology from this scheme and we have developed a non-relativistic picture which incorporates besides the confinement potential, the OGEP, the OPEP and the OSEP and with these scheme we have described both the spectra and the $N-N$ and $N-\Delta$ scattering channels [43,44]. This most naive approach is very successful.

\subsection{Constituent quarks and partons}

The models we have been discussing in the previous sections have been motivated by mostly static properties and therefore pretend to reflect the non-perturbative aspects of the theory. In what follows we would like to analyze high energy data by using them. The much spoken about success of perturbation theory is a non predictive statement. The renormalization group relates scales and therefore the success is based on experimental circumstances where either the non-perturbative aspects are cancelled between several observations, or data are fed into the theory and the evolution from one scale to another is analyzed. We would like to be predictive with the caveat that we are substituting the true theory by models. 


\subsubsection{Parton distributions from quark models}

The basic idea in this approach arises from rephrasing the OPE which states that,

$$
F_{i}^{n}\left(Q^{2}\right)=M_{i j}^{n} F_{j}^{n}\left(Q_{0}^{2}\right)
$$

i.e., the moments of structure functions at one scale are related by means of perturbatively calculable transformation matrices to the same moments at another scale [2]. If $Q_{0}^{2}$ is taken to be a low scale, what we have labelled hadronic scale, the $\mathrm{F}$ functions become highly non perturbative matrix elements in general. We substitute these matrix elements at the hadronic scale by the same matrix elements calculated with a model. In particular we are able to relate the valence quark distribution functions with the momentum distributions in the corresponding baryonic state $n_{q}^{a}$, i.e. with the hadronic wave functions of the model,

$$
x q_{V}^{a}(x)=\frac{1}{(1-x)^{2}} \int d^{3} p n_{q}^{a}(\vec{p}) \delta\left(\frac{x}{1-x}-\frac{k_{+}}{M}\right)
$$

where $a$ represents the diverse degrees of freedom (unpolarized, $\uparrow, \downarrow, \ldots$ ), $p_{+}=p_{0}-p_{z}$, $x$ is the Bjorken variable and $M$ the mass of the baryonic state.

In this way we have studied polarized and unpolarized structure function, transversity distributions and angular momentum distributions with various models [32, 33]. The results of our calculations show that these models, with the parameters fixed by low energy properties are able to provide a qualitative description of the data and therefore become predictive. They are however too naive and new ingredients not seen by the low energy probes have to be incorporated.

\subsubsection{Constituent and current quarks}

Our basic assumption above has been that gluon and sea bremsstrahlung are the source of difference between the constituents and the current quarks. We have gone beyond that scheme by incorporating some structure to the quarks following the procedure we have called ACMP [48]. Within this approach constituent quarks are effective particles made up of point-like partons (current quarks, antiquarks and gluons), interacting by a residual interaction described by a quark model [49. The structure of the hadron is obtained by a convolution of the constituent quark model wave function with the constituent quark structure function. For a proton made up of $u$ and $d$ quarks,

$$
f\left(x, \mu_{0}^{2}\right)=\int_{x}^{1} \frac{d z}{z}\left[u_{0}\left(z, \mu_{0}^{2}\right) \Phi_{u f}\left(\frac{x}{z}, \mu^{0}\right)+d_{0}\left(z, \mu_{0}^{2}\right) \Phi_{d f}\left(\frac{x}{z}, \mu^{0}\right)\right],
$$

where $\mu_{0}^{2}$ is the hadronic scale, $f=q_{v}, q_{s}, g$ (valence quarks, sea quarks and gluons respectively) and $\Phi$ represents the constituents probability in each quark and has been parametrized following general arguments of $Q C D$ as

$$
\Phi_{q f}\left(x, \mu_{0}^{2}\right)=C_{f} x^{a_{f}}(1-x)^{A_{f}-1} .
$$

The constants have been fixed by Regge phenomenology and the choice of the hadronic scale $\left(\mu_{0}=0.34 \mathrm{GeV}^{2}\right)$. The discussion can be generalized to the polarized structure functions. The procedure is able to reproduce the data extremely well and in this framework the so called spin problem does not arise. 


\section{Concluding remarks}

$Q C D$ has a complex non-perturbative realization which has escaped solution for the past 27 years. The recent progress in calculating directly from the theory has come from the lattice approximation, however these calculations are still primitive, and certainly expensive, if one wants to compare with data. In the meantime effective theories and models should provide lattice $Q C D$ with well defined scenarios where the theory can be tested.

We have emphasized the relation between models and the properties of the theory. The concept of effective theory has been most instrumental for our developments. Effective theories provide the parameters that one has to calculate from first principles. Models provide the scenarios where new physics may be envisaged.

We have approached the recent controversy, about the most adequate degrees of freedom to describe hadron properties, from the point of view of the $C C P$. Our conclusion is that there is a price to pay, namely complexity and lack of predictivity, if one doest not consider gluon degrees of freedom to address scenarios where asymptotic freedom is fundamental in determining the physics. Moreover, there is no way, besides solving $Q C D$, to describe without pions scenarios where spontaneous broken chiral symmetry dominates the dynamics. Our CCP analysis has taught us that models, called generically hybrid, are the most economical way to describe the physics of medium energy.

We have shown that many models, developed to describe low energy physics, if taken as approximate solutions of $Q C D$ in the hadronic regime, are able to describe, via evolution, properties of the asymptotic regime surprisingly well and moreover be predictive.

\section{Acknowledgements}

I have recalled some of the recent developments from the perspective of my own personal research. Many friends, luckily too many to cite them here, have accompanied me in the voyage. You may find some of them in the reference list. Others were simply too generous to sign my papers. Of all of them I would like to single out a few, because they have strongly motivated my work. Pedro Gónzalez has developed chiral quarks to its present beauty. Mannque Rho has mantained my interest in the $C C P$ and has forseen much of its power. Marco Traini has kept my faith in our early discovery: the relation between low energy models and high energy data. Sergio Scopetta has been the driving force behind our recent work in the asymptotic regime, which has confirmed our expectations. Nimai brought me back to naive models and showed me their beauty. I miss him!

\section{REFERENCES}

1. H. Fritzsch, M. Gell-Mann and H. Leutwyler, Phys. Lett B47 (1973) 365.

2. F.J. Yndurain, The theory of quark and gluon interactions (Springer Verlag, Heidelberg, Germany 1999).

3. D. Weingarten, Phys. Rev. Lett. 51 (1983) 1830; E. Witten, Phys. Rev. Lett. 51 (1983) 2351; C. Vafa and E. Witten, Nucl. Phys. 234 (1984) 173.

4. G. 't Hooft, Recent developments in gauge theories (Eds. G. 't Hooft et al, Plenum Press, New York 1980). 
5. V. Azcoiti and A. Galante, Phys. Rev. Lett. 83 (1999) 1518.

6. D.J. Gross and F. Wilczek, Phys. Rev. Lett. 30 (1973) 1323, H.D. Politzer, Phys. Rev. Lett. 13 (1973 1346.

7. R.K. Ellis, W.J. Stirling and B.R. Webber, QCD and collider physics (Cambridge University Press, Cambridge, UK 1996).

8. R.G. Roberts, The structure of the proton (Cambridge University Press, Cambridge, UK 1990).

9. G. 't Hooft, Nucl. Phys. b138 (1978) 1.

10. A. Ferrando and V. Vento, Phys. Rev. D49 (1994) 3044.

11. M. Shifman, Int. J. Mod. Phys. A14 (1999) 5017.

12. M. Creutz, Quarks, gluons and lattices (Cambridge University Press, Cambridge, UK 1983).

13. S. Mandelstam, Phys. Rep. 23 (1976) 245; G. 't Hooft, Under the spell of the gauge principle (World Scientific, Singapore 1994).

14. A. Di Giacomo, Nucl. Phys. Proc. Suppl. 47 (1996) 136.

15. N. Seiberg and E. Witten, Nucl. Phys. B426 (1994) 19, (E) B439 (1994) 485; Nucl. Phys. B431 (1994) 484.

16. T. Banks and A. Casher, Nucl. Phys. B168 (1980) 103.

17. T. Schäfer and E. V. Shuryak, Rev. of Mod. Phys. 70 (1998) 323.

18. H. Georgi, Weak interactions and modern particle physics (The Benjamin/Cummings Pub. Co., Menlo Park, CA, 1984).

19. S. Weinberg, Physica 96A (1979) 327.

20. J. Gasser and H. Leutwyler, Ann. Phys. 158 (1984) 142.

21. G. 't Hooft, Nucl. Phys. B72 (1974) 461; E. Witten, Nucl. Phys. B160 (1979) 57.

22. N. Isgur and M. Wise, Phys. Rev. D43 (1991) 819.

23. N.I. Kochelev, V. Vento and A.V. Vinnikov, Phys. Lett. B472 (2000) 247.

24. Y. Umino and V. Vento, Phys. Lett. B472 (2000) 5.

25. M. Gell-Mann, Phys. Lett. 8 (1964) 214; G. Zweig, CERN Preprints 401 and 402 (unpublished).

26. R.H. Dalitz, High energy physics (Ed. C. de Witt and M. Jacob, Gordon and Breach Science Pub., New York 1965).

27. O.W. Greenberg, Phys. Rev. Lett. 13 (1964) 598; M. Han and Y. Nambu, Phys. Rev. 139 (1965) 1006.

28. A. de Rújula, H. Georgi and S.L. Glashow, Phys. Rev. D12 (1975) 147.

29. T. DeGrand, R.L. Jaffe, K. Johnson and J. Kiskis, Phys. Rev. D12 (1975) 2060.

30. N. Isgur and G. Karl, Phys. Rev. D20 (1979) 1191, D21 (1979) 3175.

31. R.L. Jaffe, Color, spin and color dependent forces in Quantum Chromodynamics, hep-ph/001123.

32. M. Traini, L. Conci and U. Moschella, Nucl. Phys. A544 (1992) 731; M. Ropele, M. Traini and V. Vento, Nucl. Phys. A584 (1995) 634; M. Traini, A. Mair, A. Zambarda and V. Vento, Nucl. Phys. A614 (1997) 472.

33. S. Scopetta and V. Vento, Phys. Lett. B424(1998)25, Phys. Lett. B460 (1999) 8, Phys. Lett. B474 (2000) 235.

34. N.C. Mukhopadhyay and V. Vento Nucl. Phys. A643(1998) 415.

35. A. Chodos and C.B. Thorn, Phys. Rev. D12 (1975) 2733; V. Vento, M Rho, E. 
M. Nyman, J.H. Jun, G.E. Brown, Nucl. Phys. A345 (1980) 413; G.E. Brown, A.D. Jackson, M. Rho, V. Vento, Phys. Lett. B140 (1984) 285; A.W. Thomas, S. Theberge, G.A. Miller. Phys. Rev. D24(1981) 216.

36. J. Navarro and V. Vento, Phys. Lett. B140 (1984) 6, Nucl. Phys. A440 (1985) 617; Yu. A. Kuperin, A. A. Kvitsinsky, S. P. Merkurev, E. A. Yarevsky; Sov. J. Nucl. Phys. 51(1990) 141, Nucl. Phys. A523 (1991) 614.

37. S. Nadkarni, H.B. Nielsen and I. Zahed, Nucl. Phys. B253 (1985) 308.

38. B.-Y. Park, V. Vento, M. Rho and G. E. Brown, Nucl. Phys A504 (1989) 829.

39. H.-J. Lee, D.-P. Min, B.-Y. Park, M. Rho and V. Vento, Nucl. Phys. A657 (1999) 75.

40. A. Manohar and H. Georgi, Nucl. Phys. 234 (1984) 189.

41. N. Isgur, Critique of a pion exchange model for interquark forces, nucl-th/9908028.

42. L.Ya. Glozman, Reply to Isgur's 'critique of a pion exchange model for interquark forces', nucl-th/9909021.

43. A. Valcarce, P. González, F. Fernández and V. Vento, Phys. Lett. B367 (1996) 35, Phys. Rev. C61 (2000) 019803; F. Fernández, P. González and A. Valcarce, Few Body Systems Suppl. 10 (1999) 395; D.R. Entem, F. Fernández and A. Valcarce, Phys. Rev. C62 (2000) (to be published).

44. H. Garcilazo, Abstracts of this conference and to be published.

45. J. Goldstone and R.L. Jaffe, Phys. Rev. Lett. 51 (1983) 1518.

46. I. Zahed and G.E. Brown, Phys. Rep. 142 (1986) 1; M. Nowak, M. Rho and I. Zahed, Chiral nuclear dynamics (World Scientific, Singapore 1996).

47. A.D. Jackson, L. Vepstas, E. Wüst and D. Kahana, Nucl. Phys. A462 (1987) 661.

48. G. Altarelli, N. Cabbibo, L. Maiani, R. Petronzio, Nucl. Phys. B (1974) 531.

49. S. Scopetta, V. Vento and M. Traini, Phys. Lett. 421 (1998) 64; Phys. Lett. B442 (1998) 28. 